EPJ Web of Conferences 97,00013 (2015)

DOI: 10.1051 /epjconf/201597000 13

(C) Owned by the authors, published by EDP Sciences, 2015

\title{
Exotica production with ALICE
}

\author{
Benjamin Dönigus $^{1, a}$ for the ALICE Collaboration \\ ${ }^{1}$ Institut für Kernphysik, Goethe-Universität Frankfurt, Max-von-Laue-Straße 1, 60438 Frankfurt, Germany
}

\begin{abstract}
The high collision energies reached at the LHC lead to significant production yields of light (anti-)nuclei and (hyper-)nuclei in proton-proton, proton-lead and, in particular, lead-lead collisions. The excellent particle identification capabilities of the ALICE apparatus, based on the specific energy loss in the Time Projection Chamber and the velocity information in the Time-Of-Flight detector, allow for the detection of these rarely produced particles. Further, the Inner Tracking System gives the possibility to separate primary nuclei from those coming from weak decay of heavier systems. One example of such a weak decay is the measurement of the (anti-)hypertriton decay to ${ }^{3} \mathrm{He}+\pi^{-}\left({ }^{3} \overline{\mathrm{He}}+\pi^{+}\right)$. The aforementioned capabilities of the ALICE apparatus offer the unique opportunity to search for exotica, like the bound state of a $\Lambda$ and a neutron which would decay into a deuteron and a pion, or the bound state of two $\Lambda$ 's. Results on the production of stable nuclei in $\mathrm{Pb}-\mathrm{Pb}$ collisions at $\sqrt{s_{\mathrm{NN}}}=2.76 \mathrm{TeV}$ are presented, and compared with thermal model predictions. We further present the current status of the searches, by their upper limits on the production yields, and compare the results to thermal and coalescence model expectations.
\end{abstract}

\section{Introduction}

The unique particle identification capabilities of the ALICE detector [1] allow for the measurement of rarely produced exotic states created in $\mathrm{Pb}-\mathrm{Pb}$ collisions. It also gives the opportunity to search for hypothetical states. In particular we study anti-matter, such as light anti-nuclei and anti-hypernuclei, and search for states like the H-dibaryon, a hexaquark state (uuddss), which was already predicted in 1977 [2] using a bag model calculation. Recent lattice QCD calculations [3, 4] also suggest a bound state, with binding energies in the range $13-50 \mathrm{MeV} / c$. A chiral extrapolation of these lattice calculations to a physical pion mass resulted in a H-dibaryon which is unbound by either $13 \pm 14$ $\mathrm{MeV} / \mathrm{c}$ [5] or it lies close to the $\Xi \mathrm{p}$ threshold [6]. This renewed the interest in the experimental searches for the H-dibaryon which were ongoing since its prediction.

The energy regime reached at the LHC leads to large production probabilities of such particles, as described for example by thermal models [7-9]. The chemical freeze-out temperature $T$, the volume $V$ and the baryo-chemical potential $\mu_{\mathrm{B}}$ are the only three free parameters in this approach as long as one assumes an equilibrated system. To account for possible non-equilibrium effects the factors $\gamma_{s}$ and $\gamma_{q}$ are very often introduced (e.g. [9]). An open question in the field of heavy-ion collisions is whether the production of nuclei and other multi-baryon states, such as hypernuclei or also hyper-matter, is

\footnotetext{
a e-mail: benjamin.doenigus@cern.ch
} 


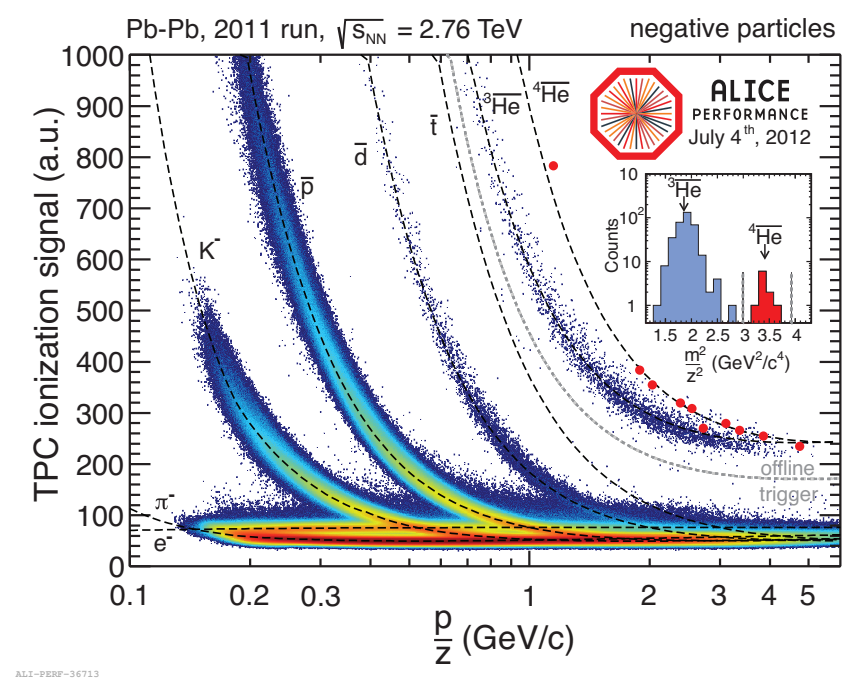

Figure 1. TPC $\mathrm{d} E / \mathrm{d} x$ spectrum for negative particles after a selection of events that contain at least one ${ }^{3} \overline{\mathrm{He}}$ or ${ }^{4} \overline{\mathrm{He}}$ candidate. The inlet shows the $\mathrm{m}^{2} / \mathrm{z}^{2}$ distribution for this pre-selected data. The 10 anti-alphas clearly identified by TPC and TOF are indicated as red dots.

entirely described by a thermal model or if those objects are formed by coalescence of hadrons or even directly from coalescence quarks.

\section{Anti-Alpha}

The excellent performance of the Time Projection Chamber (TPC) [10] and the Time-Of-Flight detector (TOF) [1] allows for the clear identification of charged stable particles over a range of 0.15 to $5 \mathrm{GeV} / c$ in rigidity $R=p / z$, where $p$ is the track momentum and $z$ is the charge number. Combining the specific energy loss $(\mathrm{d} E / \mathrm{d} x)$ in the TPC and the the velocity measurement in the TOF, anti-alpha nuclei were identified.

Figure 1 shows the $\mathrm{d} E / \mathrm{d} x$ versus rigidity distribution for candidates after the offline selection for negative particles in the region where the bands of ${ }^{3} \overline{\mathrm{He}}$ and ${ }^{4} \overline{\mathrm{He}}$ are clearly visible. Below a rigidity of $p / z \approx 2 \mathrm{GeV} / c$ three candidates are clearly identified based on the $\mathrm{d} E / \mathrm{d} x$ information only. At higher $p / z$ the energy-loss information of the candidates is combined with mass determination performed with the TOF detector following the relation $m^{2} / z^{2}=R^{2} /\left(\gamma^{2}-1\right)$. The inlet in Figure 1 shows the $m^{2} / z^{2}$ distribution for all tracks within a $2 \sigma$-band around the expected $\mathrm{d} E / \mathrm{d} x$ for ${ }^{4} \overline{\mathrm{He}}$. The 10 identified anti-alphas are highlighted in both the $m^{2} / z^{2}$ and the $\mathrm{d} E / \mathrm{d} x$ versus rigidity plot.

Since the measurement is limited by statistics careful studies have been done to understand the signal counts and the background components mainly coming from ${ }^{3} \overline{\mathrm{He}}$ which is misidentifed as ${ }^{4} \overline{\mathrm{He}}$ in the region of the TPC where their Bethe-Bloch bands overlap or where issues with the track matching between TPC and TOF is causing misidentification.

The acceptance $\times$ efficiency was estimated using a Monte Carlo simulation where once the transport through the detector material was done with a patched version (to take care of anti-nuclei) of GEANT3 and once with GEANT4 to account for differences in the absorption modeling. The difference was taken into account as a substantial part of the systematics. 


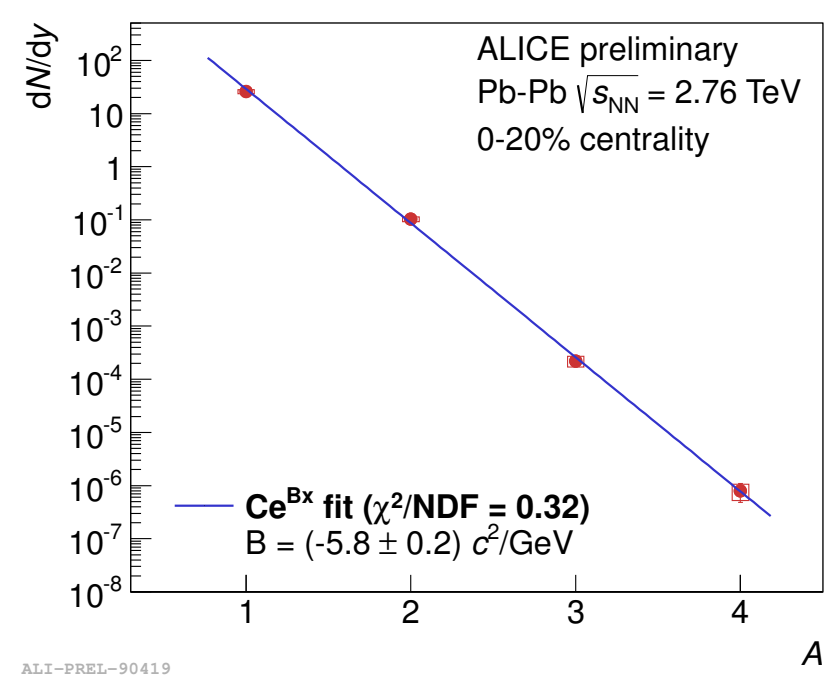

Figure 2. Production yields of protons, deuterons, ${ }^{3} \mathrm{He}$ and anti- ${ }^{4} \overline{\mathrm{He}}$ as function of mass number A. The line indicates an exponential fit.

The corrected $\mathrm{d} N / \mathrm{d} y$ value is shown together with the other light nuclei in Figure 2 as function of the mass number $A$.

\section{Deuteron}

The measurement of deuterons is affected by a huge background, as for all nuclei, coming from the interaction of primary particles with detector material. This is not relevant for the measurement of anti-nuclei, like the anti-alpha (see Section 2). Rejection of this background is possible by restricting the Distance-of-Closest-Approach (DCA) of the track to the primary vertex in z-direction $\mathrm{DCA}_{Z}$ and then fitting the $\mathrm{DCA}_{X Y}$ distribution to extract the signal in the $p_{\mathrm{T}}$ window between 0.6 and $1.9 \mathrm{GeV} / c$ (this technique is described in more detail in [11]).

The deuterons are identified using only the $\mathrm{d} E / \mathrm{d} x$ measurement in the TPC for $p_{\mathrm{T}}<1.5 \mathrm{GeV} / c$ and combining the TPC and TOF information for higher $p_{\mathrm{T}}$. A sample of deuteron selected with a 3 $\sigma$ cut around the expected $\mathrm{d} E / \mathrm{d} x$ value is initially used to build the $m^{2}-m_{\mathrm{d}}^{2}$-distribution, where $m$ is the mass measured with TOF and $m^{2}$ is calculated as described in section 2 . Then, this distribution is fitted with a Gaussian function + exponential tail in $p_{\mathrm{T}}$ intervals. The extracted yields are then efficiency and acceptance corrected and the final spectra are shown in Figure 4 for five different collision centralities. The spectra show a characteristic hardening with increasing centrality, qualitatively similar to proton spectra. To extract $p_{\mathrm{T}}$ integrated yields the spectra in the different centrality bins are fitted by individual blast-wave distributions.

\section{Hypertriton}

The hypertriton ${ }_{\Lambda}^{3} \mathrm{H}$ is the lightest known hyper-nucleus and is composed by a proton, a neutron and a $\Lambda$. Its mass is $2.991 \pm 0.002 \mathrm{GeV} / c^{2}$ and it has a lifetime comparable with the one of the free $\Lambda$, whereas its branching ratio is not well known. 

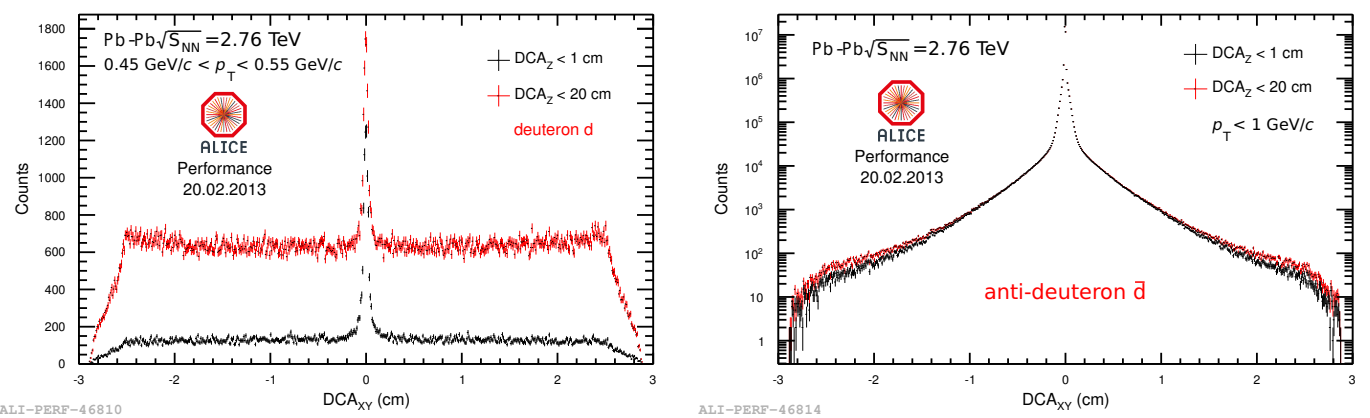

Figure 3. $\mathrm{DCA}_{X Y}$ distribution for different $\mathrm{DCA}_{Z}$ values for deuterons (left) and anti-deuterons (right). The anti-deuterons are shown in a logarithmic scale to show the small differences visible at larger ${ }^{D C A_{X Y}}$ values, which would otherwise not be seen.

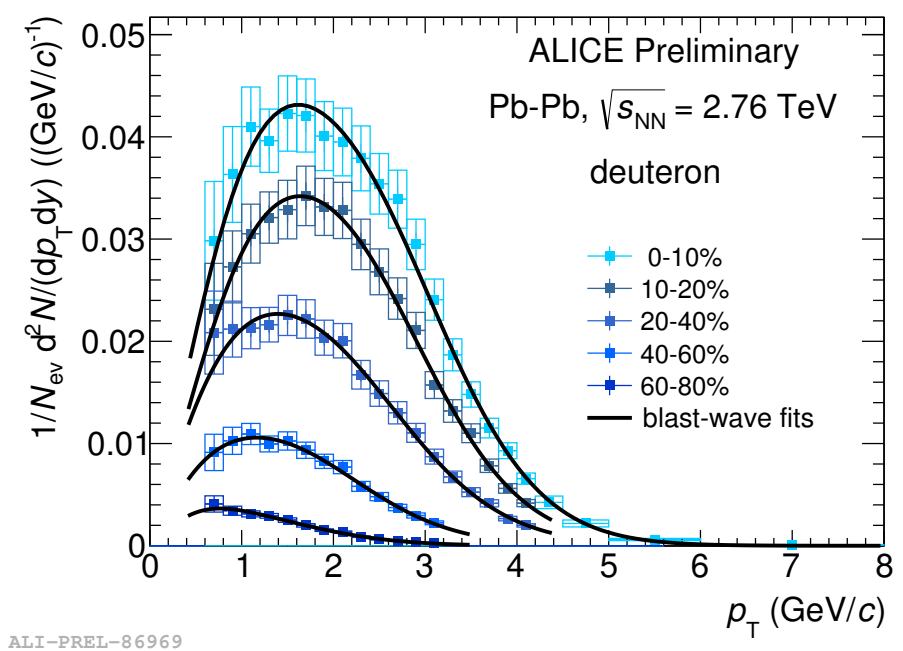

Figure 4. Measured deuteron spectra at $\sqrt{s_{\mathrm{NN}}}=2.76 \mathrm{TeV}$ for five different centralities. The lines through the measured points are individual blast-wave fits to extrapolate towards low $p_{\mathrm{T}}$.

Hypertriton and anti-hypertriton are identified via their weak decays $\left({ }_{\Lambda}^{3} \mathrm{H} \rightarrow{ }^{3} \mathrm{He}+\pi^{+}\right.$and $\frac{3}{\Lambda} \overline{\mathrm{H}} \rightarrow{ }^{3} \overline{\mathrm{He}}+\pi^{-}$), applying topological selection criteria and particle identification as discussed before. In the same sample as used for the anti-alpha analysis (see Section 2) a signal for hypertriton (anti-hypertriton) with a significance of 4.6 (2.6) has been obtained. The background was evaluated with two different methods, i.e. like-sign and a combined fit (Gaussian on top of a third order polynomial, where the Gaussian describes the signal and the polynomial the background shape), shown in Figure 5.

The raw yield has been extracted from the invariant mass distribution and corrected for acceptance $\times$ efficiency, similar to the anti-alpha measurement discussed in Section 2. From this the $\mathrm{d} N / \mathrm{d} y \times$ 


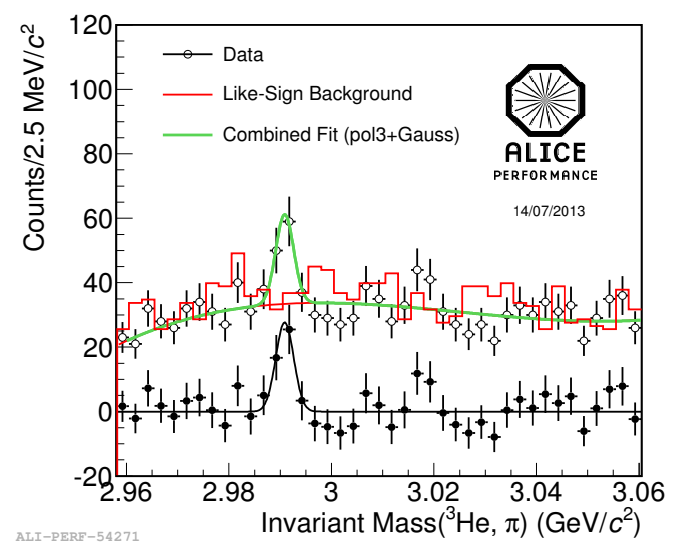

Figure 5. Invariant mass of ${ }^{3} \mathrm{He}+\pi^{-}$and ${ }^{3} \overline{\mathrm{He}}+\pi^{+}$pairs in semi-central (10-50\%) events.

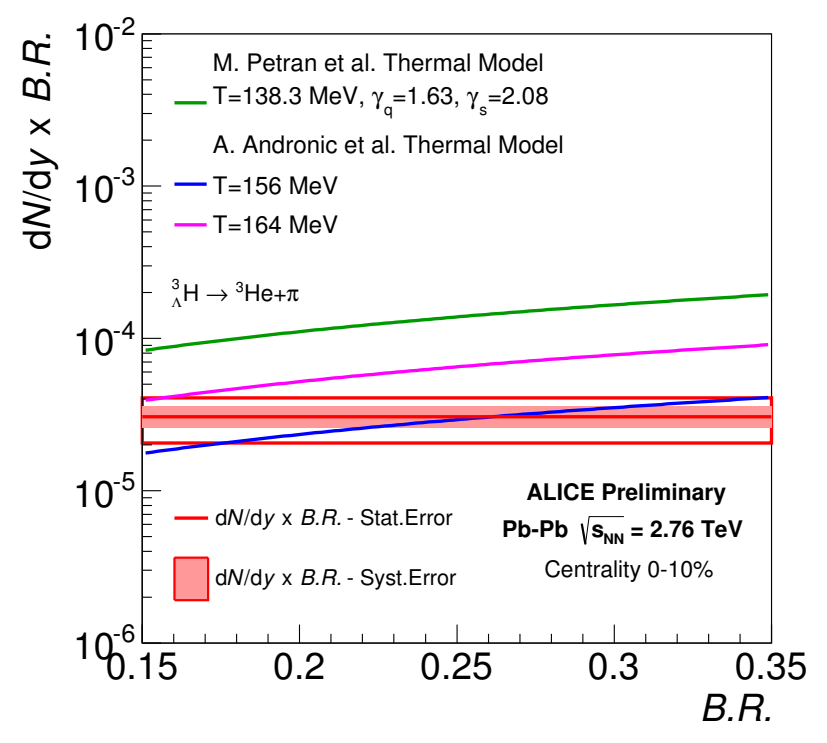

Figure 6. $\mathrm{d} N / \mathrm{d} y \times$ branching ratio (B.R.) of the hypertriton compared to different models.

branching ratio has been determined, which is shown in Figure 6, where it is compared to different thermal model expectations. The best description of the data is given by the equilibrium thermal model at a temperature of $156 \mathrm{MeV}$. At this value the theory curve hits the experimental value at around $25 \%$ for the branching ratio. 

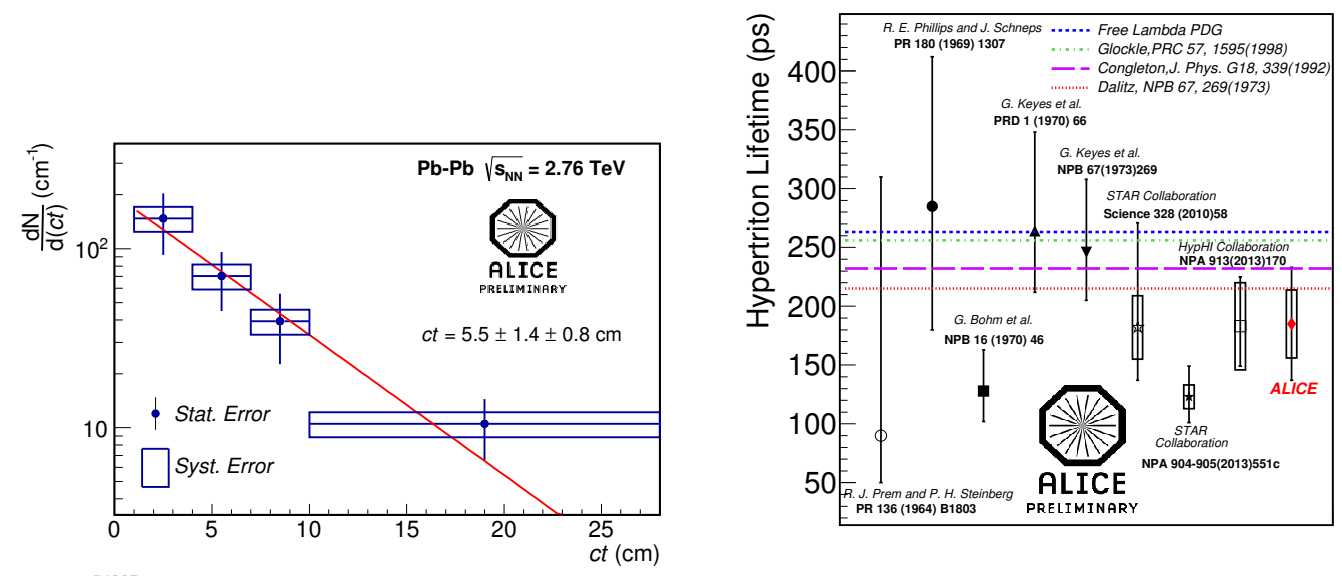

Figure 7. Combined hypertriton and anti-hypertriton $c$ t bins, fitted with an exponential function (left panel). Compilation of the available measurements of the hypertriton lifetime, the ALICE point is highlighted in red.

The available statistics allows for the lifetime measurement of the hypertriton (sum of particle and anti-particle) in four $c t$ bins (displayed in the left part of Figure 7). From a fit of the data a lifetime of the ${ }_{\Lambda}^{3} \mathrm{H}$ was determined which is in good agreement with the world average as visible from Figure 7.

\section{Search for the $\Lambda \mathrm{n}$ bound state and the H-dibaryon}

For the H-dibaryon we investigated the $\Lambda \mathrm{p} \pi$ decay. Other possible decay channels contain a neutron which is difficult to detect with the ALICE setup. The expected branching ratios depend on the binding energy, as shown in [12]. Since a low binding energy is favoured by the current theoretical considerations - if bound at all - we concentrate on the mass region of $2.2-2.3 \mathrm{GeV} / c^{2}$ in the decay channel $\Lambda \mathrm{p} \pi$. In this channel a signal for a bound state would result in a peak in the invariant mass or in a broad structure above the $\Lambda \Lambda$ threshold in case of a resonant state. In a similar way, we also study here the possible decay of a $\Lambda \mathrm{n}$ bound state decaying into $\mathrm{d}+\pi^{-}$which was observed at GSI by the HypHI collaboration [13] at a mass of $2.054 \mathrm{GeV} / c^{2}$.

The results shown here for the H-dibaryon and the $\overline{\Lambda n}$ bound state are based on the analysis of about 13.8 million $\mathrm{Pb}-\mathrm{Pb}$ events in the centrality class of $0-80 \%$ taken with the ALICE apparatus in 2010. The reconstructed invariant mass distributions are shown in Figure 8 for the H-dibaryon and in Figure 9 for the $\overline{\Lambda n}$. No evidence for a signal, neither for the H-dibaryon nor the $\overline{\Lambda n}$ bound state was found. The Figures 8 and 9 also show the expected signal, for the H-dibaryon for two assumed masses of $2.21 \mathrm{GeV} / c^{2}$ and $2.23 \mathrm{GeV} / c^{2}$ (corresponding to binding energies of $21 \mathrm{MeV} / c^{2}$ and $1 \mathrm{MeV} / c^{2}$ ) and a possible $\overline{\Lambda n}$ signal. We focus here on the $\overline{\Lambda n}$, since the background is much lower compared to $\Lambda \mathrm{n}$. The expected signal was computed estimating the acceptance $\times$ efficiency (from a Monte Carlo simulation), the production rates as predicted by the thermal-model [14] and the expected branching ratios [12, 15]. For the Monte Carlo simulation, involving full decay kinematics and transport in the material utilizing GEANT3, the lifetime of the free $\Lambda$ hyperon was assumed for both exotic states. 


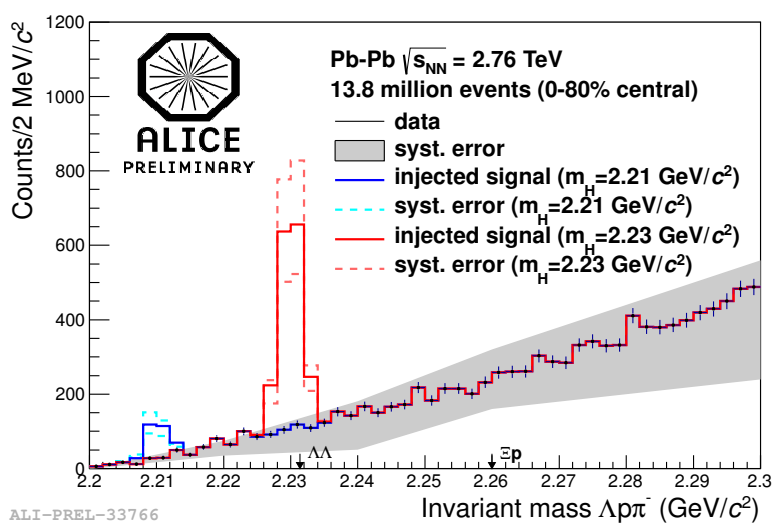

Figure 8. Invariant mass distribution of $\Lambda \mathrm{p} \pi$. The expected signal is shown for two cases, in red a slightly bound $\mathrm{H}$-dibaryon ( $1 \mathrm{MeV}$ binding energy) and in blue a H-dibaryon bound by $20 \mathrm{MeV}$.

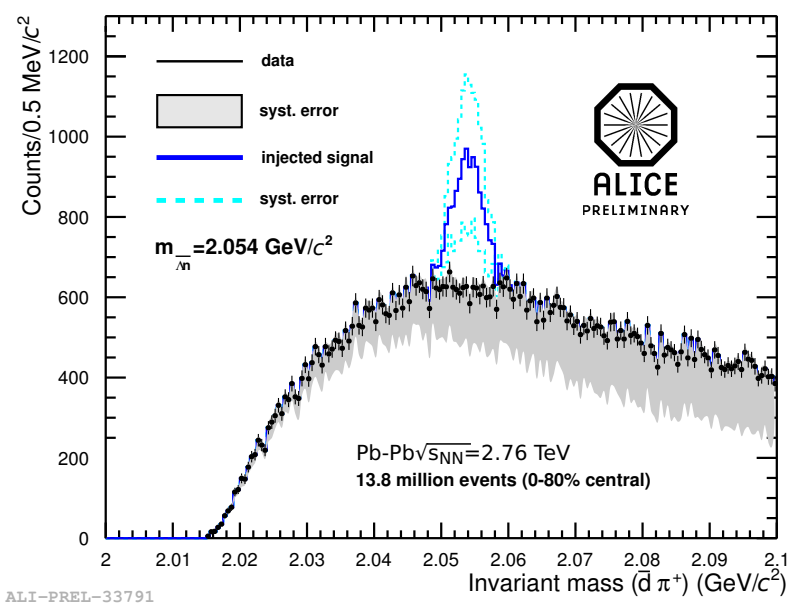

Figure 9. Invariant mass distribution of anti-deuterons and positive pions. The expected signal is shown in blue.

\section{Summary and Conclusion}

The measured yield of the anti-alpha, the deuteron and the hypertriton presented here compare well with the current best equilibrium thermal model fit [16] which gives a temperature of about $156 \mathrm{MeV}$. One can now contrast the upper limits set for the exotica currently under investigation by ALICE, i.e. $\mathrm{H}$-dibaryon and $\Lambda \mathrm{n}$ bound state, with different models. Figure 10 shows a comparison of the upper limits to the equlibrium thermal model [14] (164 MeV, which was the prediction for LHC and 156 $\mathrm{MeV}$, the current best fit), the non-equilibrium thermal model prediction [17] from their current best fit [9] and coalescence predictions (quark and hadron coalescence) from the ExHIC collaboration [18]. All models are at least a factor 10 above the upper limits. Currently an effort continues to further 


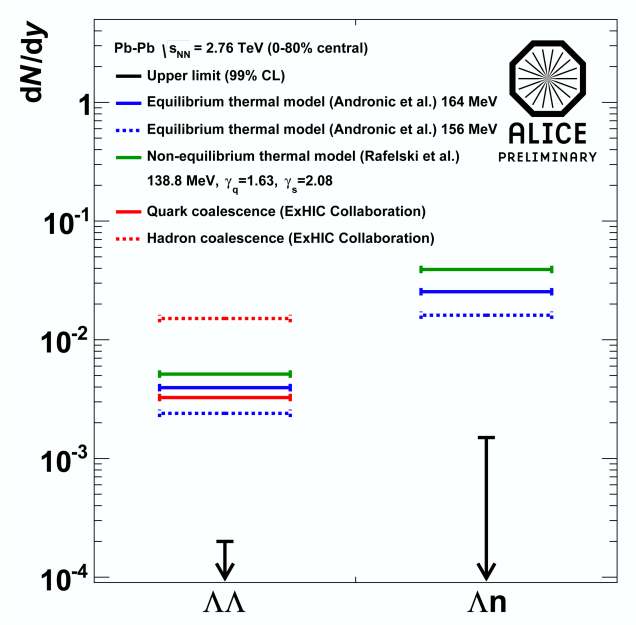

Figure 10. Upper limits of production yields for H-dibaryon $(\Lambda \Lambda)$ and $\Lambda \mathrm{n}$ bound state compared with different models.

constrain these upper limits by utilising more statistics than was previously used. This would allow for a more stringent conclusion concerning the existence of these exotic states.

\section{References}

[1] K. Aamodt et al. (ALICE Collaboration), JINST 3, S08002 (2008)

[2] R. Jaffe, Phys. Rev. Lett. 38, 195 (1977); erratum ibid 38, 617 (1977)

[3] S. R. Beane et al., Phys. Rev. Lett. 106, 162001 (2011)

[4] T. Inoue et al., Phys. Rev. Lett. 106, 162002 (2011)

[5] P. E. Shanahan, A. W. Thomas, R. D. Young, Phys. Rev. Lett. 107, 092004 (2011)

[6] J. Haidenbauer, U.-G. Meißner, Phys. Lett. B 706, 100 (2011)

[7] A. Andronic et al., Phys. Lett. B 697, 203 (2011)

[8] J. Cleymans et al., Phys. Rev. C 84, 054916 (2011)

[9] M. Petráň et al., Phys. Rev. C 88, 034907 (2013)

[10] J. Alme et al., Nucl. Instr. Meas. A 622, 316 (2010)

[11] B. Abelev et al. (ALICE Collaboration), Phys. Rev. C 88, 044910 (2013)

[12] J. Schaffner-Bielich, R. Mattiello, H. Sorge, Phys. Rev. Lett. 84, 4305 (2000)

[13] C. Rappold et al. (HypHI Collaboration), Phys. Rev. C 88, 041001 (2013)

[14] A. Andronic, private communication

[15] J. Schaffner-Bielich, private communication

[16] M. Floris, Nucl. Phys. A 931, 103 (2014)

[17] M. Petráň, private communication

[18] S. Cho et al. (ExHIC Collaboration), Phys. Rev. C 84, 064910 (2011) 